\title{
Kai Zen und die Kunst, ärztliche Qualität
}

\section{zu managen}

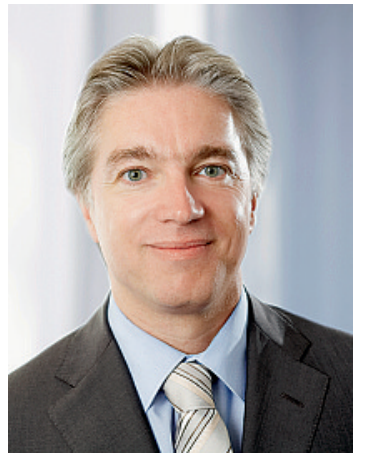

Daniel Herren
Kai Zen ist ein Managementbegriff aus Japan. In der freien Übersetzung bezeichnet der Begriff die Veränderung (Kai) zum Besseren (Zen) und bezeichnet damit eine Philosophie, die im Gegensatz zum Versuch der sprunghaften Veränderung den Ansatz in der ständigen, schrittweisen Verbesserung sucht. Schön und gut, werden Sie sagen, was hat das aber mit ärztlicher Leistungserbringung zu tun? Beim genaueren Hinschauen eben einiges. Der Qualitätsbegriff, wie er momentan von vielen Akteuren interpretiert wird, ist geprägt von klassisch statischen Vorstellungen.

Darin dienen Q-Messungen zwar dazu, einen Ist-Zustand aufzuzeigen, bieten aber keine Hand für Problemlösungen. Zum Beispiel bringt die Angabe einer Mortalitätsziffer zu einem spezifischen Eingriff nur dann etwas, wenn dieser Indikator erstens relevant für diesen Eingriff ist (quantitativ wie qualitativ) und zweitens sich die Möglichkeit bietet, sich in diesem Indikator zu verbessern. Genau dort setzt Kai Zen an. In einem ständig laufenden Zyklus wird in kleinen Schritten versucht, einerseits zu

\section{Der momentane Qualitätsbegriff ist geprägt von klassisch statischen Vorstellungen}

erhalten, was gut ist, und andererseits eine kontinuierliche Verbesserung in Problemzonen anzustreben. Bezogen auf unsere laufenden Diskussionen um die Messung der ärztlichen Leistungserbringung würden wir gut daran tun, etwas mehr Kai Zen und etwas weniger Kennzahlenarithmetik zu berücksichtigen.

Qualitätsmessungen sollen einer Logik folgen, die mit dem Erfassen von Qualitätsaktivitäten beginnt und diese dann würdigt und bewertet. Erst wenn die sinnvollen und nutzenorientierten Aktivitäten für die jeweilige Leistungserbringergruppe bestimmt sind und die Messme- thodik eindeutig feststeht, kann man Resultate evaluieren. Für die FMH ist es wichtig, bereits in dieser Phase der Evaluation die Koproduzenten einzubeziehen. Darauf basierend können schliesslich Massnahmen bestimmt werden, die die Qualität nachhaltig verbessern. Dies können

\section{Bezogen die Messung der ärztlichen Leistungserbringung, würden wir gut daran tun, etwas mehr Kai Zen und etwas weniger Kennzahlenarithmetik zu berücksichtigen}

Belohnungen, auch nichtfinanzieller Art, sein, die die Leistungserbringer motivieren, auch weiterhin überdurchschnittliche Leistungen zu vollbringen, es können Hilfestellungen für Leistungserbringer sein, die dem Bereich Qualität zu wenig Beachtung schenken und dort einen Nachholbedarf aufweisen. Ein anreizorientiertes System darf deshalb nicht direkt bei den Massnahmen einsetzen, sondern muss diesen ganzen Prozess, analog der Idee im Kai Zen, durchlaufen.

Darauf basiert unsere Idee des Incentive-for-QualityModells. Dieses hat in dieser Hinsicht das Potential, den Risiken der Fehlanreize und Fehlversorgung, die oft mit finanziellen Anreizsystemen einhergehen, entgegenwirken zu können.

Dr. med. Daniel Herren MHA, Mitglied des Zentralvorstandes der FMH, Verantwortlicher für das Ressort DDQ

1 Pirsig RM. Zen und die Kunst ein Motorrad zu warten. Frankfurt: Fischer; 1974.

2 Imai M. Kaizen. Der Schlüssel zum Erfolg der Japaner im Wettbewerb. München: Langen-Müller; 1992.

3 Hutwelker R. hard und soft facts zur Prozessverbesserung. 2005. www.activepartner.de $\rightarrow$ Prozesse \& Ressourcen $\rightarrow$ KAIZEN/KVP $\rightarrow$ Materialien $\rightarrow$ Prozessverbesserungen mit KAIZEN/ KVP (Artikel) 